\title{
Peertechz
}
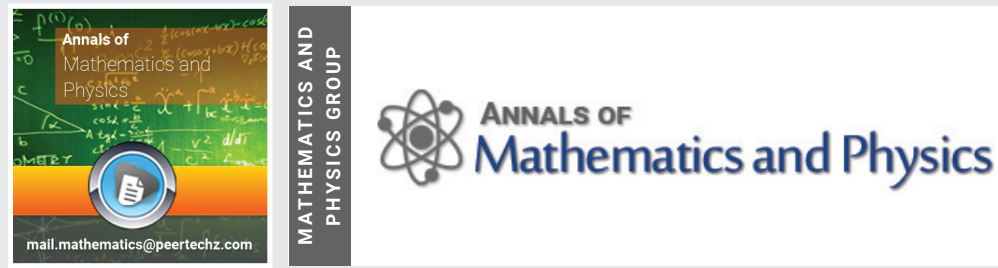

Review Article

\section{Analysis of the axial stability for an assembly of optical modes with stochastic}

\section{fluctuations type Markov chain}

Received: 30 February, 2020

Accepted: 11 May, 2020

Published: 12 May, 2020

*Corresponding author: G Martinez Niconoff, Departamento de Óptica, Instituto Nacional de Astrofísica Óptica y Electrónica, Luis Enrique Erro N. 1, 72840 Tonantzintla, Puebla, México, E-mail: gmartin@inaoep.mx

https://www. peertechz.com

Check for updates

\section{Martinez Vara P1, Gutierrez Ojeda C², A Garcia Guzman², E Saldivia Gomez ${ }^{2}$, G Arenas Muñoz ${ }^{2}$, JC Atenco Cuautle ${ }^{2}$ and G Martinez Niconoff ${ }^{2 *}$}

${ }^{1}$ Facultad de Ingienería, Benemérita Universidad Autónoma de Puebla, Av. San Claudio s/n, 72570 Puebla, Puebla, México

²Departamento de Óptica, Instituto Nacional de Astrofísica Óptica y Electrónica, Luis Enrique Erro N. 1, 72840 Tonantzintla, Puebla, México

\begin{abstract}
We describe the engineering of optical modes whose axial structure follows fluctuations of Markov-chain-type. These stochastic processes are associated with a sequence of time subintervals of duration $\Delta T$. Each subinterval is linked to a Bessel mode of integer order selected according to a Markov-chain-type process. The resulting optical field is implemented using computational simulations for Markov-chain-type Ehrenfest. This process was chosen because it models the thermodynamic equilibrium and it is related with the evolution and stability of optical systems. The matrix representation for the stochastic process allows to incorporate entropy properties for the calculation of the purity of the optical field.
\end{abstract}

\section{Introduction}

This manuscript appears due the necessity to generate tunable optical tweezers for trapping particles generating asymmetric distributions. This is performed using a temporal ensemble of non-diffracting beams. The study is supported by the fact that arbitrary optical field can be expressed as a sum of coherent modes [1], where the mode representation satisfies an Fredholm integral equation whose kernel is the autocorrelation function $\Gamma\left(t_{1}, t_{2}\right)$ [2], implying that the coherence degree is time depending. The generic features of the optical field can be identified only when the optical processes under study presents a stationary behavior, and the integral equation acquires the form of a convolution function. However, a large variety of optical fields do not satisfy stationary properties [3,4]. One example can be found, in the statistical analysis of a process with few photons. The statistical properties display a probability distribution similar to that in a queuing process [5]. Another example is the propagation of light trough atmosphere [6] . In the present manuscript, we describe the engineering of optical modes, whose amplitude function is described by a stochastic succession of elementary modes type Markov chain. The mean irradiance distribution is characterized by using the time depending purity function, whose temporal evolution is related with the entanglement described by the transition probabilities associated to the modes succession. The stochastic process is a type convergent Markov chain, that reaches a final equilibrium configuration $[7,8]$. The time depending optical field offers interesting applications in cryptographic information, dynamic holography, tunable spectroscopy and self-healing processes. The theoretical model consists in associating a stochastic matrix to the chain. The stochastic matrix elements are related to the transition probabilities among possible states. The dynamics of the process is analyzed by interpreting the matrix as a transformation applied to a random vector, that represents the initial state of the chain. The evolution of the initial state is 
obtained by sequentially applying the transition matrix, which generates a final stochastic matrix named $\mathrm{N}$-step stochastic matrix [5] which takes information from the probability of initial states reaching a final state in $\mathrm{N}$ steps.

A fundamental point consists in the analysis of the stability of the process. In order to do so, we studied processes whose $\mathrm{N}$-step stochastic matrix acquires a regular form, this means that all the matrix elements are different from zero. This assures the stability of the Markovian process. The issues to be addressed are the following: for a given process Markovtype chain and assuming an initial state which has associated an initial random vector, it is necessary to identify how this vector evolves generating entanglement among the elements of the resulting random vector. The final state is described by calculating the entropy values implicit in the $\mathrm{N}$-step stochastic matrix $[9,10]$. From this analysis we are able to determinate the time evolution of the purity of the Markovian optical field $[11,12]$. To maintain a geometrical point of view, we take advantage on the fact that each Markovian chain type process is associated with a directed graph named digraph, where each physical state corresponds to a node. The evolution of the process generates connectivity among the nodes, corresponding to the entanglement of the process. To associate an optical meaning, the nodes are matched with Bessel modes of integer order and the Markovian chain corresponds to the evolution of the connectivity among the modes. The global structure of the resulting optical field is obtained when the entropy and purity values reach a stable configuration.

\section{Description of markovian chains}

A stochastic process is a parametrized set of randomvariables. We consider the time as the parameter i.e. $X(t), i$ $=1,2, . ., n$. The stochastic process is completely determined by the nth-order correlation function expressed as

$$
\begin{aligned}
P\left(x_{0}, x_{1}, \ldots, x_{n}\right) & =P\left(x_{n} \mid x_{n-1}, \ldots, x_{1}\right) P\left(x_{n-1}, x_{n-2}, \ldots, x_{1}, x_{0}\right) \\
& =P\left(x_{n} \mid x_{n-1}, \ldots, x_{1}\right) P\left(x_{n-1} \mid x_{n-2}, \ldots, x_{1}\right) P\left(x_{n-2}, \ldots, x_{1}\right) \ldots P\left(x_{1} \mid x_{0}\right),
\end{aligned}
$$

where the subindex refers to time and $P\left(x_{0}, x_{1}, x_{2}, \ldots\right)$ is the occurrence probability of the random variables $\left(x_{0}, x_{1}, x_{2}, \ldots\right) . P$ $\left(x_{n} \mid, x_{n-1}, x_{n-2}, \ldots\right)$ is the conditional probability that represents the probability of occurrence of $x_{n}$ given the occurrence of an event defined by $\left(X_{n-1}, X_{n-2}, \ldots\right)$. When the process depends on its recent history, which is known as the Markovian hypothesis, Eq.

(1) acquires a simplified form as follows:

$P\left(x_{0}, x_{1}, \ldots, x_{n}\right)=P\left(x_{n} \mid x_{n-1}\right) P\left(x_{n-1} \mid x_{n-2}\right) \ldots P\left(x_{1} \mid x_{0}\right) P\left(x_{0}\right)$.

The previous expression defines the Markov chain where the term $P\left(x_{\mathrm{i}} \mid x_{\mathrm{i}-1}\right)$ is known as the transition probability. To analyze the evolution of the Markov chain, we associate to the process a stochastic matrix representation, which is interpreted as a transformation of the random initial vector given by

$$
\vec{\pi}_{0} \mathbf{P}=\left(\begin{array}{lll}
a_{0} & \cdots & a_{n}
\end{array}\right)\left(\begin{array}{cccc}
P_{00} & P_{01} & \cdots & P_{0 n} \\
P_{10} & P_{11} & \cdots & P_{1 n} \\
\vdots & \vdots & \ddots & \vdots \\
P_{n 0} & P_{n 1} & \cdots & P_{n n}
\end{array}\right)=\vec{\pi}_{1},
$$

where $\pi_{0}=\left(a_{0}, \ldots, a_{\mathrm{n}}\right)$ is the random initial vector and $P_{\mathrm{ij}}$ is the probability of the random variable to pass from the i state to the $\mathrm{q}$ state with the following property:

$\sum_{q} P_{i q}=1, \quad i=1,2, \ldots, n$.

The Markovian process is obtained by applying recursively the matrix to the resulting vector, i.e.

$\vec{\pi}_{1}=\vec{\pi}_{0} \mathrm{P}, \quad \vec{\pi}_{2}=\vec{\pi}_{1} \mathrm{P}=\vec{\pi}_{0} \mathrm{P}^{2}, \quad \ldots \quad \vec{\pi}_{N}=\vec{\pi}_{0} \mathrm{P}^{N}$

where stochastic matrix $\mathbf{P}^{\mathrm{N}}$ is known as the $\mathrm{N}$-step transition matrix. Very important properties of the $\mathrm{N}$-step transition matrix can be highlighted. When this matrix is applied on an initial state, forbidden states can appear. A final state cannot be reached from this initial state, this effect is closely related to bifurcation properties. Another effect consists in the chaos generation, which consists on small changes on the probability values of the initial states that evolve toward states completely different. The N-step matrix allows the identification of the equilibrium states, which occur when the entropy values are stabilized. All of the properties previously mentioned depend on the specific Markovian chain. For this reason, in the following section we show a case when the N-step matrix reaches a stable configuration. The evolution of entropy values and the connection among the nodes can be identified by associating to the $\mathrm{N}$-step stochastic matrix to a digraph of the form sketched in Figure 1.

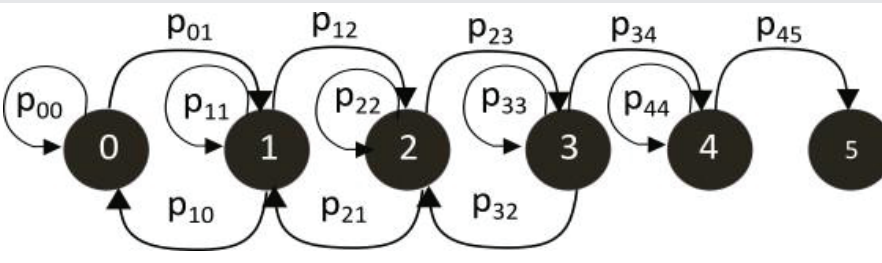

Figure 1: Digraph associated to a Markov type process.

The recursively application of the stochastic matrix is related to the evolution of the digraph. It can be easily identified from the digraph that the $\mathrm{N}$-step stochastic matrix acquires a regular form and then the process reaches a stable configuration. Thus, the elements in the $\mathrm{N}$-step stochastic matrix are different from zero, this property fulfills for a Markovian chain type Ehrenfest. Moreover, this type of process is applied in order to

Citation: Martinez Vara P, Gutierrez Ojeda C, Guzman AG, Gomez ES, Niconoff GM, et al. (2020) Analysis of the axial stability for an assembly of optical modes with stochastic fluctuations type Markov chain. Ann Math Phys 3(1): 011-016. DOI: https://dx.doi.org/10.17352/amp.000012 
describe the thermodynamic equilibrium, which allows to be matched with the stability of optical processes. The recursively application of the stochastic matrix is related to the evolution of the digraph. It can be easily identified from the digraph that the $\mathrm{N}$-step stochastic matrix acquires a regular form and then the process reaches a stable configuration. Thus, the elements in the $\mathrm{N}$-step stochastic matrix are different from zero, this property fulfills for a Markovian chain type Ehrenfest. Moreover, this type of process is applied in order to describe the thermodynamic equilibrium, which allows to be matched with the stability of optical processes.

\section{Markov chain-type ehrenfest process}

To describe the Markov chain-type Ehrenfest process, we can conveniently use a box model as follows. We assume two boxes labeled as $A$ and $B$ that contain $n$ balls. Box $A$ contains balls, and box $B$ contains $n-q$ balls. The balls are labeled from 1 to $n$ and they are randomly distributed in each box. The Ehrenfest process consists of selecting one ball and transferring it to the other box with probability $\alpha$, or letting it remain in the same box with probability $1-\alpha$. Keeping this idea

in mind, we can easily show that the stochastic matrix is

$$
E=\left(\begin{array}{ccccccc}
(1-\alpha) & \alpha & 0 & 0 & 0 & \cdots & 0 \\
\frac{\alpha}{n} & (1-\alpha) & \left(\frac{n-1}{n}\right) \alpha & 0 & 0 & \cdots & 0 \\
0 & \frac{2 \alpha}{n} & (1-\alpha) & \left(\frac{n-2}{n}\right) \alpha & 0 & \cdots & 0 \\
0 & 0 & \frac{3 \alpha}{n} & (1-\alpha) & \left(\frac{n-3}{n}\right) \alpha & \cdots & 0 \\
\vdots & \vdots & \vdots & \vdots & \vdots & \ddots & \vdots \\
0 & 0 & 0 & 0 & \cdots & \alpha & (1-\alpha)
\end{array}\right) .
$$

To obtain a better understanding of the Ehrenfest process evolution, a numerical example for $n=4$ and $\alpha=1 / 2$ is performed. The issues discussed are: how the row values are transformed and how the matrix converges after $N$ steps. The following matrix expressions, show the resulting stochastic matrix after 1, 2 and 25 steps.

$$
\begin{gathered}
E=\left(\begin{array}{cccc}
0.5000 & 0.5000 & 0 & 0 \\
0.1250 & 0.5000 & 0.3750 & 0 \\
0 & 0.2500 & 0.5000 & 0.2500 \\
0 & 0 & 0.5000 & 0.5000
\end{array}\right), E^{2}=\left(\begin{array}{cccc}
0.3125 & 0.5000 & 0.1875 & 0 \\
0.1250 & 0.4063 & 0.3750 & 0.0938 \\
0.0313 & 0.2500 & 0.4688 & 0.2500 \\
0 & 0.1250 & 0.5000 & 0.3750
\end{array}\right), \\
\cdots, E^{25}=\left(\begin{array}{llll}
0.0714 & 0.2857 & 0.4286 & 0.2143 \\
0.0714 & 0.2857 & 0.4286 & 0.2143 \\
0.0714 & 0.2857 & 0.4286 & 0.2143 \\
0.0714 & 0.2857 & 0.4286 & 0.2143
\end{array}\right) .
\end{gathered}
$$

From the last matrix expression, we can identify that all elements in each column have the same value, which corresponds to the final equilibrium state [8]. The structure of Eq. (7) shows that the product of a row in the arbitrary random vector with an $\mathrm{N}$-step stochastic matrix reproduces the matrix row. This represents the equilibrium of the process that is nondependent on the initial random vector. From this equilibrium condition, we can deduce some generic features, particularly the maximum entanglement, that is related to the succession of the digraphs shown in Figure 2. The entanglement features correspond to the evolution of the different states of the probability values among the nodes. This analysis implies the evolution of the entropy values. It must be noted that the changes in the assigned probability values of the initial stochastic matrix, implies the modification of the connectivity between states. This information becomes evident on table of Figure 2-d, where all nodes show the same connectivity among states.

\section{Generation of ehrenfest optical modes}

In this section, we implement the Ehrenfest process in the optical context. To perform this, we conveniently start to define an optical mode as a solution to the Helmholtz equation in the following form [13]:

$$
\phi(x, y, z)=f(x, y) \exp (i \beta z),
$$

the function $f(x, y)$ satisfies the eigenvalue equation

$$
\nabla_{\perp}^{2} f(x, y)+K^{2} f(x, y)=\beta^{2} f(x, y),
$$

that propagates along the $z$ coordinate. Eq. (9) can be solved using polar coordinates. This allows to easily identify the solutions as a set of Bessel modes of an integer order given by (Figure 2).

$$
\left\{e^{i \beta z} J_{n}(2 \pi r d) e^{i n \theta}\right\} n=0, \pm 1, \pm 2, \ldots
$$

We remark that all of the modes have the same phase function along the $\mathrm{z}$ coordinate. Being this a condition of the modes that present diffraction-free features. In the appendix we describe the condition for an optical field to correspond with an optical mode. using this representation, we propose as definition for a stochastic mode a sequence of modes whose structure follows a stochastic process and locally presenting diffraction free features, a particular case occurs when integer order Bessel modes are selected following a Markov chain type process.

Eq. (10) can be matched with the box model of the Ehrenfest process described in the previous section. By replacing the label in each ball by $J_{0}, J_{1}, \ldots, J_{n}$ we describe the evolution of initial state $x_{0}=\left(a_{0}, \ldots, a_{n}\right)$. This vector corresponds to the coordinates that represent the appearance of the mode with the following interpretation: Assuming that the process has time duration $T$, divided by $n$ subintervals of length $\Delta T$. In each subinterval, a Bessel mode of integer order is selected. Thus, the optical field consists of a succession of mode-type chains where the occurrence of the ith Bessel mode is $n \alpha_{i}$. With this interpretation, the optical field assumes the structure shown in Fig. 3. A liquid crystal display (LCD) is implemented to generate the boundary condition that consists of an annular slit angularly modulated for synthetizing the corresponding

Citation: Martinez Vara P, Gutierrez Ojeda C, Guzman AG, Gomez ES, Niconoff GM, et al. (2020) Analysis of the axial stability for an assembly of optical modes with stochastic fluctuations type Markov chain. Ann Math Phys 3(1): 011-016. DOI: https://dx.doi.org/10.17352/amp.000012 
Bessel mode [13,14]. The structure of the chain corresponds to the Ehrenfest mode.

To get an understanding the evolution of the Markovian mode, it is convenient to describe a tree graph, shown in Fig. 4. The dotted line represents the sequence $\left(\mathrm{J}_{0}-\mathrm{J}_{0}-\mathrm{J}_{1}-\mathrm{J}_{2} \ldots\right)$, and the dotted arrowed line represents $\left(J_{0}-J_{1}-J_{2}-J_{1} \ldots\right)$. From this representation we generate time structured modes. All of them must exhibit the same irradiance mean when the equilibrium is reached. In the early steps the corresponding modes displays different irradiance values. This is shown in Figure 5 that was obtained with MATLAB software.

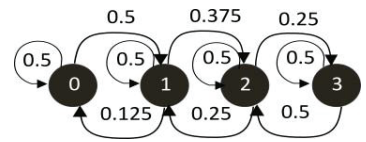

a)

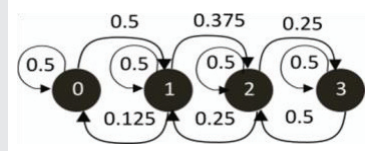

c)

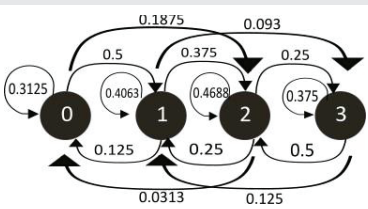

b)

\begin{tabular}{c|c|c|c|c} 
& 0 & 1 & 2 & 3 \\
\hline 0 & 0.0714 & 0.2857 & 0.4286 & 0.2143 \\
1 & 0.0714 & 0.2857 & 0.4286 & 0.2143 \\
2 & 0.0714 & 0.2857 & 0.4286 & 0.2143 \\
3 & 0.0714 & 0.2857 & 0.4286 & 0.2143
\end{tabular}

d)
Figure 2: Depicture of the stochastic matrix process corresponding to Eq. (7). a) Digraph associated to the initial stochastic matrix. b) Digraph for $E^{2}$.c) Digraph for $E^{25}$ when the process reaches a stablish configuration. d) Numerical representation of the digraph shown in c).

Figure 5. The mean irradiance after N-steps for an Ehrenfest type process. a) Represents the initial state associated to a $J_{0}$ Bessel mode. The initial probability vector is irradiance after 2 -steps, the probability vector is $(1 / 2,1 / 2,0,0)$. c) Mean irradiance after 25-steps, the probability vector is $(0.0714,0.2857,0.4286,0.2143)$. It must be noted that this last vector corresponds with a row for the stabilized N-step stochastic matrix. The expression for the mean irradiance can be related to the probability vector as $I={ }^{(N)} n\left(P_{0}^{2} J_{0}^{2}+P_{1}^{2} J_{1}^{2}+P_{2}^{2} J_{2}^{2}\right)$, where ${ }^{(N)} n P_{i}$ is the occu number for the irradiance of each mode.

The Ehrenfest mode consists of a sequence of Bessel modes of integer order where each sequence appears according to a certain probability value. The state starts with a zero order Bessel beam, whose irradiance is shown in Fig 5-a, this state evolves following the chain and after 3 steps, the initial vector evolves toward the vector whose irradiance is given by $\alpha_{0}^{(2)} J_{0}^{2}+\alpha_{1}^{(2)} J_{1}^{2}+\alpha_{2}^{(2)} J_{2}^{2}$. When the experiment is performed $n-$ times, the number of occurrences of each element of the basis can be obtained from the transformed random vector. The irradiance mean after 3 steps is shown in Fig 5-b. When the number of steps increases, the chain is stabilized and the irradiance mean distribution is shown in Figure 5-c. We analyzed the global optical fields once the equilibrium is reached. The mean irradiance distribution presents diffraction free features. The later is easily understood because the irradiance associated to each mode is non-depending on the $z$-coordinate. However, locally, these fields follow a sequence of optical modes according to the Markov chain selected. The resulting optical mode consist in a sequence of time changing blocks that do not follow a stationary process. For this reason, the mode is characterized using the entropy models in the following section.

\section{Entropy, purity and interference between Markovian modes}

From the fact that a Markovian process has associated a stochastic matrix, we can identify the generic features through entropy calculation. This allows to describe the mode's structural properties. We proposed the calculus of the Von Neumann entropy [10] in order to obtain the entropy value from the N-step stochastic matrix. The entropy is calculated from the principal diagonal elements. The resulting value acts as a reference value for the entropy measurement obtained from the elements of the secondary diagonal. This value contains information about the correlation among the constitutive modes [15]. By comparing these entropy values, we can deduce how the correlation function evolves, allowing to understand the irradiance distribution as a function of $\mathrm{N}$, which represents the number of applications of the initial stochastic matrix. The

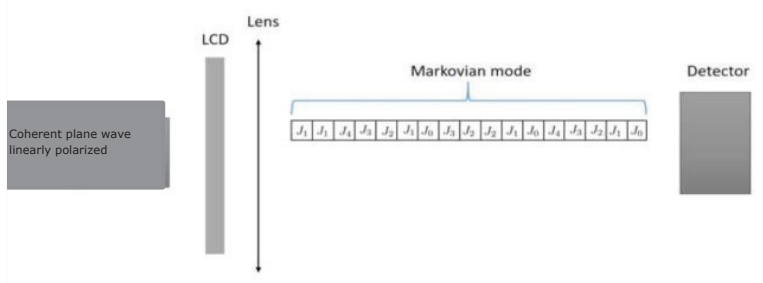

Figure 3: To generate the Bessel modes, we illuminate a LCD containing an annular slit with time-dependent angular modulation with a coherent plane wave linearly polarized.

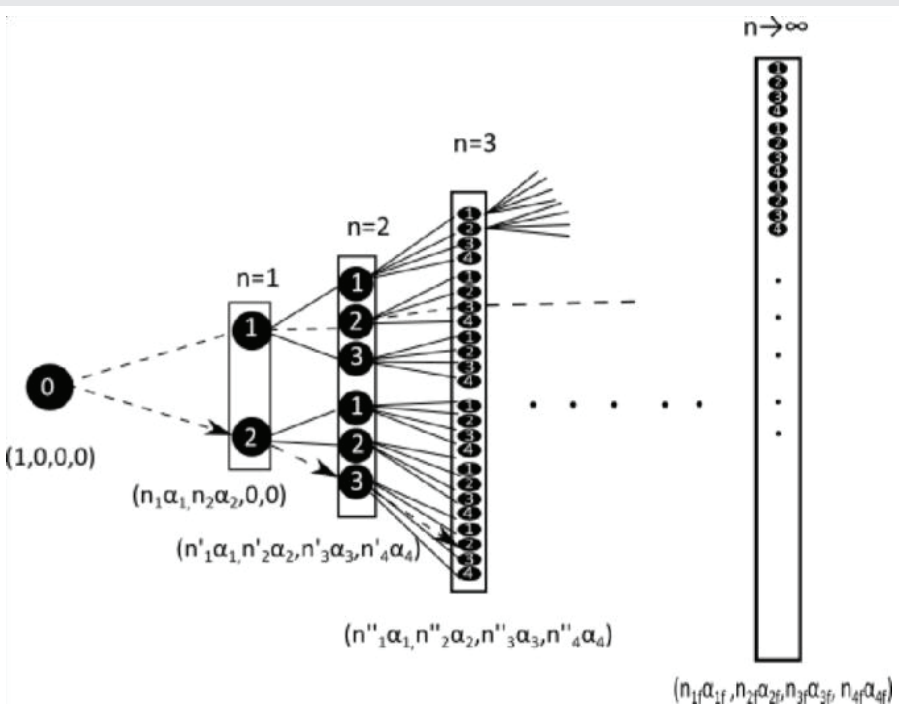

Figure 4: Sketch of the entanglement between states as " $n$ " grows, during this evolution is possible see that the probability density is distributed between the accessible states

Citation: Martinez Vara P, Gutierrez Ojeda C, Guzman AG, Gomez ES, Niconoff GM, et al. (2020) Analysis of the axial stability for an assembly of optical modes with stochastic fluctuations type Markov chain. Ann Math Phys 3(1): 011-016. DOI: https://dx.doi.org/10.17352/amp.000012 

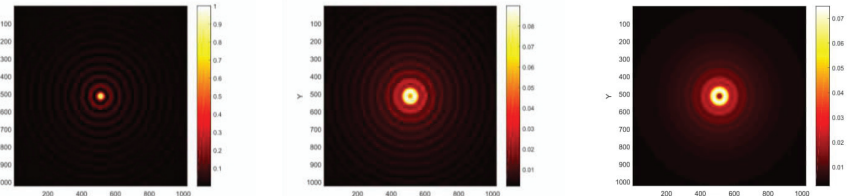

Figure 5: The mean irradiance after $\mathrm{N}$-steps for an Ehrenfest type process. a) Represents the initial state associated to a $J 0$ Bessel mode. The initial probability vector is $(1,0,0,0)$. b) Mean irradiance after 2-steps, the probability vector is $(1 / 2$, $1 / 2,0,0)$. c) Mean irradiance after 25 -steps, the probability vector is $(0.0714,0.2857$, $0.4286,0.2143)$. It must be noted that this last vector corresponds with a row for the stabilized Nstep stochastic matrix. The expression for the mean irradiance can be related to the probability vector as $I={ }^{(N)} n\left(P 0^{2} J 0^{2}+P 1^{2} J 1^{2}+P 2^{2} J 2^{2}\right)$, where ${ }^{(N)} n P i$. is the occurrence number for the irradiance of each mode.

Von Neumann entropy is defined as

$$
S_{v}=-\operatorname{Tr}\left(E^{N} \operatorname{Ln} E^{N}\right)
$$

where $\operatorname{Tr}$ denotes the trace of stochastic matrix $E^{\mathrm{N}} \operatorname{Ln} E^{\mathrm{N}}$. It must be noted that this type of entropy contains information for the irradiance distribution. However, we need to describe the entanglement among the elements of the basis. This description can be obtained by proposing the correlation entropy calculus as an ansatz using the elements in the secondary diagonal. This entropy takes the following form:

$$
S_{c}=-\operatorname{Tr} D\left(E^{N} \operatorname{Ln} E^{N}\right),
$$

where $\operatorname{TrD}$ denotes the trace of the secondary diagonal. Then, a good method of describing the mode structure is applied by calculating the difference of the entropy values, which is expressed as

$$
\Delta S=S_{c}-S_{v} .
$$

We propose this definition, since it can easily prove that the correlation entropy is always lower-bounded by the Von Neumann entropy $S_{\mathrm{c}}, \ldots S_{\mathrm{v}}$. To compare the entropy values, each diagonal needs to satisfy the normalization condition. From Eq. (13), certain interesting cases can be identified. The limit case occurs when $\Delta S=0$. Its physical meaning is that all irradiance events involved during the process participate in the global irradiance distribution. The other case occurs when $\Delta S$ $=S$. There is no interaction among the elements of the basis; thus, they are statistically independent. However, this case is not permitted in the Markov chain-type Ehrenfest process. Finally, more entropy measurements can be obtained from the $q$ th-row elements, expressed as

$$
{ }_{q} S_{f}=-\sum_{i} \alpha_{i q} \operatorname{Ln}\left(\alpha_{i q}\right)
$$

where $\alpha_{\mathrm{iq}}$ denotes the elements in the $q$ th row and satisfies ${ }_{\mathrm{i}}$ $\alpha_{\mathrm{iq}}=1$ for $q=0,1,2, \ldots, n$. From this entropy row, an order relationship is easily identified, e.g.,

$$
S_{q}<S_{2}=S_{4}<S_{5}<\ldots
$$

this means that a $q$ th-Bessel mode appears in a principal manner, followed by the second- order Bessel mode that appears in the same proportion as the fourth-order Bessel modes, and so on. From this order relationship, we can associate a purity measurement to the Ehrenfest mode [12] as follows:

$$
P_{q}=1-\frac{S_{q}^{(n)}}{\sum_{l=0}^{N} S_{i}^{(n)}}
$$

which determines the similitude of the Markovian mode with the $J_{\mathrm{q}}$ mode because $\sum_{q=0}^{n} P_{q}=1$. The entropy values for the Ehrenfest mode are given by $S_{\mathrm{v}}=S_{\mathrm{c}}=0.5383$. The equality indicates that the process reaches an equilibrium condition. In addition, all of the rows have the same entropy value. Consequently, from the purity definition, we can deduce that the resulting mode is the same for each element of the basis. This result is expected because the Ehrenfest process describes an equilibrium system. Consequently, once the equilibrium condition is reached and as a result of the isotropy of the process, all the basis elements appear in the same proportion. The purity concept describes the times each element appears in a given mode.

\section{Conclusions}

We proposed a theoretical model to generate stochastical optical modes with a set of Bessel modes ordered according to a Markovian-chain type process. The model was obtained by associating a stochastic matrix to this process and recursively applying it to an initial state obtaining an $\mathrm{N}$-step stochastic matrix. The latter represents the probability for an initial state to reach a final state in $\mathrm{N}$-steps. This matrix also takes information of the process evolution, obtained through entropy values calculated from the rows which have a random vector structure. The set of entropy values allowed us to associate a purity degree for the resulting mode giving information about the entanglement of the basis elements. Computational simulations were performed with MATLAB software for a Markov chain-type Ehrenfest process. This type process was implemented because it describes the conditions under which a thermodynamic process reaches equilibrium. The optical field evolves towards an optical mode which presents diffraction free features. The proposed model can be implemented to analyze interesting features of the optical field such as interference effects by means of the purity concept. This is possible assuming a set of Markovian modes, all of them with the same purity value. This means that the interference can be described by counting the number of coincidences among the basis elements, and can be applied to generate tunable holography. Other immediate applications are cryptographic transference information, entanglement of arbitrary optical fields, selfhealing analysis [16-21] and tunable optical tweezers more details can be found in [22-25].

(Appendix) 


\section{References}

1. Ostrovsky AS, Romero O, Martínez-Niconoff G, Ramirez-San-Juan J (2001) On the modal representation of partially coherent imagery. Revista Mexicana de Fisica 47: 404-407. Link: https://bit.ly/2YSEx2e

2. Mandel L, Wolf E (1995) Optical Coherence and Quantum Optics. Link: https://bit.ly/3bnlHI2

3. Bogolubov NN, Aliskenderov El, Shumovsky AS, Quang T (1987) Statistical properties of photons in collective resonance fluorescence. J Phys B: At Mo Phys 20: 1885-1890. Link: https://bit.ly/2Lja0m0

4. Krantz DH (1975) Color measurement and color theory: I. representation theorem for grassmann structures. J Math Psychol 12: 283-303. Link: https://bit.ly/2zrGdVA

5. Hoel SPH, Stone C (1972) Introduction to Sthochastic Processes.

6. Vaseva I, Fedoruk M, Rubenchik A, Turitsyn S (2016) Light self-focusing in the atmosphere: Thin window model. Sci Rep 6: 30697. Link: https://bit.ly/2YSxarm

7. Niconoff GM, Rodriguez MAT, Gonzalez GD, Vara PM, Xique RS, et al. (2015) Markovian optical modes. Opt Lett 40: 863-866. Link: https://bit.ly/3clM11F

8. Chen YP (2000) Which design is better? ehrenfest urn versus biased coin. Adv Appl Probab 32: 738-749. Link: https://bit.ly/3copl0x

9. Balaji S, Mahmoud HM, Watanabe O (2006) Distributions in the ehrenfest process. Stat and Probab Lett 76: 666-674. Link: https://bit.ly/2Af7P0x

10. Barakat $R$, Brosseau $C$ (1993) Von neumann entropy of $n$ interacting pencils of radiation. J Opt Soc Am A 10: 529-532. Link: https://bit.ly/2YQR469

11. Moya-Cessa H, Moya-Cessa JR, Landgrave J, Martínez-Niconoff G, Perez Leija A, et al. (2008) Degree of polarization and quantum-mechanical purity. J Eur Opt Soc Rapid Publ 3: 08014. Link: https://bit.ly/2YPi3Po

12. Picozzi A (2004) Entropy and degree of polarization for nonlinear optical waves. Opt Lett 29: 1653-1655. Link: https://bit.ly/2LlxOWg

13. Durnin J (1987) Exact solutions for nondiffracting beams. J Opt Soc Am A 4 651-654. Link: https://bit.ly/2WkUU5N

14. Martínez-Niconoff G, Martínez-Vara $P$, Andrés-Zarate $E$, Silva-Barranco J Munoz-Lopez J (2013) Synthesis of sources with markovian features. J Eur Opt Soc Rapid publications 8. Link: https://bit.ly/2A9RABQ

15. Selvamuthu D, Di Crescenzo A, Giorno V, Nobile A (2015) A continuous-time ehrenfest model with catastrophes and its jump-diffusion approximation. $J$ Stat Phys 161: 326-345. Link: https://bit.ly/2ztZkyc

16. Sapozhnikov O (2012) An exact solution to the helmholtz equation for a quasigaussian beam in the form of a superposition of two sources and sinks with complex coordinates. Acoust Phys 58: 41-47. Link: https://bit.ly/2SSZpCm

17. Kotlyar VV, Kovalev AA, Soifer VA (2014) Asymmetric bessel modes. Opt Lett 39: 2395-2398. Link: https://bit.ly/3cyezoo

18. Barnett SM, Phoenix SJ (1989) Entropy as a measure of quantum optical correlation. Phys Rev A 40: 2404-2409. Link: https://bit.ly/2WiVEbD

19. Jones PH, Maragò OM, Volpe G (2015) Optical Tweezers: Principles and Applications. Link: https://bit.ly/2AhHv65

20. Wang F, Chen Y, Lina G, Liu L, Cai Y (2017) Complex gaussian representations of partially coherent beams with nonconventional degrees of coherence. J Opt Soc Am A 34: 1824-1829. Link: https://bit.ly/3dyAYCb

21. Janousek J, Morizur JF, Treps N, Lam PK, Harb C, et al. Optical entanglement of co-propagating modes. Nat Photonics 3: 399-402. Link: https://go.nature.com/3dDdvQf

22. Pang Y, Gordon R (2012) Optical trapping of a single protein. Nano Lett 12 402-406. Link: https://bit.ly/2WInFIO

23. Cao T, Qiu Y (2018) Lateral sorting of chiral nanoparticles using fanoenhanced chiral force in visible region. Nanoscale 10: 566-574. Link: https://bit.ly/2Ac4FdT

24. Hester B, Campbell GK, López-Mariscal C, Filgueira CL, Huschka R, et al. (2012) Tunable optical tweezers for wavelength-dependent measurements Rev Sci Instruments 83: 043114. Link: https://bit.ly/2YQzU8P

25. Teeka C, Jalil MA, Yupapin PP, Ali J (2010) Novel tunable dynamic tweezers using dark-bright soliton collision control in an optical add/drop filter. IEEE Transactions on NanoBioscience 9: 258-262. Link: https://bit.ly/3fwU2CR

\title{
Discover a bigger Impact and Visibility of your article publication with
} Peertechz Publications

\author{
Highlights \\ * Signatory publisher of ORCID \\ * Signatory Publisher of DORA (San Francisco Declaration on Research Assessment) \\ - Articles archived in worlds' renowned service providers such as Portico, CNKI, AGRIS, \\ TDNet, Base (Bielefeld University Library), CrossRef, Scilit, J-Gate etc. \\ * Journals indexed in ICMJE, SHERPA/ROMEO, Google Scholar etc. \\ - OAI-PMH (Open Archives Initiative Protocol for Metadata Harvesting) \\ * Dedicated Editorial Board for every journal \\ * Accurate and rapid peer-review process \\ * Increased citations of published articles through promotions \\ * Reduced timeline for article publication \\ Submit your articles and experience a new surge in publication services \\ (https://www.peertechz.com/submission).
}

Peertechz journals wishes everlasting success in your every endeavours.

Copyright: @ 2020 Martinez Vara P, et al. This is an open-access article distributed under the terms of the Creative Commons Attribution License, which permits unrestricted use, distribution, and reproduction in any medium, provided the original author and source are credited.

Citation: Martinez Vara P, Gutierrez Ojeda C, Guzman AG, Gomez ES, Niconoff GM, et al. (2020) Analysis of the axial stability for an assembly of optical modes with stochastic fluctuations type Markov chain. Ann Math Phys 3(1): 011-016. DOI: https://dx.doi.org/10.17352/amp.000012 